\title{
'Mayan Kisin', a High-yielding, Red-fruited, Habanero-type Hot Pepper (Capsicum chinense Jacq.) Cultivar
}

\author{
Nancy Santana-Buzzy ${ }^{1}$, Adriana Canto-Flick, and Eduardo Balam-Uc \\ Unidad de Bioquímica y Biologia Molecular de Plantas, Centro de Investigación \\ Científica de Yucatán, Calle 43 No. 130, Chuburná de Hidalgo, Mérida, Yucatán, \\ CP 97200, México
}

\section{Marta Alvarez Gil \\ Dpto. Genética y Mejoramiento de Plantas, Instituto Nacional de Ciencias Agrícolas (INCA), gaveta postal 1, San José de las Lajas, Mayabeque, CP 32700, Cuba}

Additional index words. capsaicinoids, germoplasm, pepper, variety

The Center of Scientific Research of Yucatan (CICY) has released a new openpollinated cultivar of habanero pepper: Mayan Kisin. This is distinguished by its high performance, its bright red and very spicy fruit. 'Mayan Kisin' originated from four selection cycles carried out on landraces collected in the region of Valladolid, in the state of Yucatan, Mexico. Although the center of origin of Capsicum chinense is located in South America, in the area located between southern Brazil and eastern Bolivia, western Paraguay and northern Argentina (Aguilar-Rincón et al., 2010), the Yucatan Peninsula is recognized as a center of genetic diversity of the species $C$. chinense Jacq. (Latournerie et al., 2001). In this region there are orange, red, yellow, and purple fruits of this species. It is said that the habanero pepper grown in the Yucatan Peninsula can be distinguished from habanero peppers grown in the region of the world, by its peculiar aroma, taste, and more particularly, its high level of pungency. Probably, the combination of soil and climate in the Yucatan Peninsula, located in the Mexican southeast, confers the habanero pepper cultivated in Yucatán Peninsula with characteristics that set it apart from habanero peppers cultivated in any other region of the world. These attributes allowed it to qualify for the $D e$ nomination of Origin Habanero Pepper of the Yucatan Peninsula, published by the Official Gazette of the Federation (DOF: 4 June 2010). Paradoxically, the cultivars of habanero, cultivated in the region, are characterized by their low yields, small and orange fruits, as well as the high variability

\footnotetext{
Received for publication 19 Nov. 2015. Accepted for publication 16 Dec. 2015.

This publication is an output from a research project funded by FOMIX-YUC (Fondos Mixtos of Yucatan) and CONACYT.

We acknowledge the Ministry of Foreign Affairs of Mexico (SRE) for the fellowship support to Marta Alvarez Gil.

${ }^{1}$ Corresponding author. E-mail: buzzy@cicy.mx.
}

in their populations. Nonetheless, there is a growing demand for the habanero pepper produced in this region, both in the national and international markets. Obtaining habanero pepper cultivars with high fruit quality, high yield, and pungency, has become imperative for those trying to improve their cultivation. Thus, a program for the improvement of the product is in development, based on the variability of landraces conserved in the Yucatán Peninsula, from which 'Mayan Kisin' was selected. 'Mayan Kisin' is a novelty for the fresh market because of its high productivity and pungency, the size of the fruit, and its bright red color. It is a multipurpose cultivar for its high potential for the industry and the export. Mayan Kisin is the first cultivar of habanero pepper with red color fruit that has been registered in the National Catalogue of Vegetables Varieties National System of Inspection and Seed Certification (SNICS), in Mexico.

\section{Origin}

Mayan Kisin is an open-pollinated cultivar of the species $C$. chinense, obtained by individual selection within an improvement program developed in the CICY, from landraces of the region, collected in the municipality of Valladolid, in the state of Yucatan. The aim of this work was to select a productive, pungent, red fruit cultivar, destined for the food industry.

Habanero pepper samples were collected in different localities of the Yucatan Peninsula throughout 2001-04. This germplasm is conserved in a work collection in which the diversity of the habanero pepper existing in the Yucatan Peninsula is represented. In this collection were catalogued 30 plants of red fruit. A population with the descendants of these 30 plants was established in the field (17 descendants per each plant were planted per row) to select elite plants by individual selection. Taking into consideration the high variation present in the population generated, it was designed a strategy of individual selection (four cycles) as from the progeny of the elite plants. The first two cycles were performed in the field conditions applying a selection pressure of $20 \%$ and $15 \%$, respectively. The other two cycles of selection were performed $(10 \%$ and $2 \%$ selection pressure) and developed under greenhouse conditions, to avoid crosspollination between progenies of different plants ("line"). In each cycle of selection, the seeds of each plant were extracted from ripe fruits and conserved individually. The following characters were used as the selection criteria: color of fruit (red), number of loculi (3-4), length and diameter of the fruit, days to flower, weight of fruit, and number of fruits per plant. As a result of the selection processes, nine promissory "lines" were selected. Subsequently the marketable yield (ripe and unripe fruits) and pungency test were performed in the nine promissory "lines". A randomized design (20 plants per "line") with four repetitions was established in 2011, 2012, and 2013 under greenhouse conditions. The average yield was estimated from the fruits harvested in 6-8 months. A commercial cultivar of open pollination, Habanero, produced by the company SEMINIS, was used as control. From the results of the yield test and pungency analyses, the "line" BVII was chosen to overcome the cultivar control. BVII is also a "line" of early flowering, bright-red fruits, high productivity, and high pungency (670, 325 SHU). BVII was registered by the SNICS in the year 2010 as 'Mayan Kisin' (Registry number CP-141). The name "Kisin" means "devil," in the Mayan language. This denomination was given for two reasons: as a tribute to the ancient Mayan culture and because of the high pungency of its fruit. This cultivar was propagated by seed under isolation in greenhouse conditions for distribution to farmers in the region.

\section{Description}

The most outstanding attribute of the new cultivar is its ability to produce high yields of red, pungent fruits. The mean results of three replicated trials conducted under greenhouse conditions in Chicxulub Pblo., Yucatan, in 2011-13 showed that the yield of marketable fruit (multiple harvests) from the 'Mayan Kisin' (3.67 kg/plant) exceeded the yield of marketable fruit harvested from the 'Habanero' (2.9 kg/plant) (Table 1). The average number of fruits/plant was 266; mean fruit weight $(13.8 \mathrm{~g})$, average width $(3.2 \mathrm{~cm})$, and average length $(5 \mathrm{~cm})$ of the fruit significantly surpassed the mean weight $(9.9 \mathrm{~g})$, width $(2.7 \mathrm{~cm})$, and length $(4.2 \mathrm{~cm})$ of fruits from the control cultivar (Table 1). Although only the yield test data under greenhouse conditions are shown in Fig. 1A-F, the productivity of the Mayan Kisin cultivar (A) in the field, (B) in hydroponics, and (C) in greenhouse is presented in both mature and immature fruit status (Fig. 1D-F). 'Mayan Kisin' is characterized by the high content of capsaicinoids in its fruit (670,325 SHU), considerably higher than the control cultivar (150,678 SHU) (Table 1). The quantification of capsaicinoids was performed following the method reported 
Table 1. Yield and fruit characteristics of the open-pollinated cultivar Mayan Kisin and the commercial cultivar SEMINIS grown in three greenhouse trials in 2011, 2012, and $2013 .{ }^{2}$

Fruit characteristics

Cultivars Fruits/plant Yield kg/plant Fruit wt $(\mathrm{g})$ Length $(\mathrm{cm})$ Width $(\mathrm{cm})$ Scoville units (SHU)

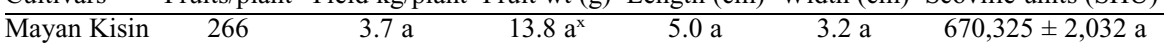

\begin{tabular}{lllllll} 
Habanero $^{w}$ & 292 & $2.9 \mathrm{~b}$ & $9.9 \mathrm{~b}$ & $4.2 \mathrm{~b}$ & $2.7 \mathrm{~b}$ & $150,678 \pm 3,711 \mathrm{~b}$ \\
\hline
\end{tabular}

${ }^{2}$ Four repetitions per trial (20 plants per repetition).

${ }^{y}$ The data are shown with the mean value \pm SE.

${ }^{x}$ Means within a row followed by the same letter are not significantly different at $P \leq 0.05$, by Tukey's Studentized range test.

wOpen-pollinated control cultivar (Seminis Seeds, CA).
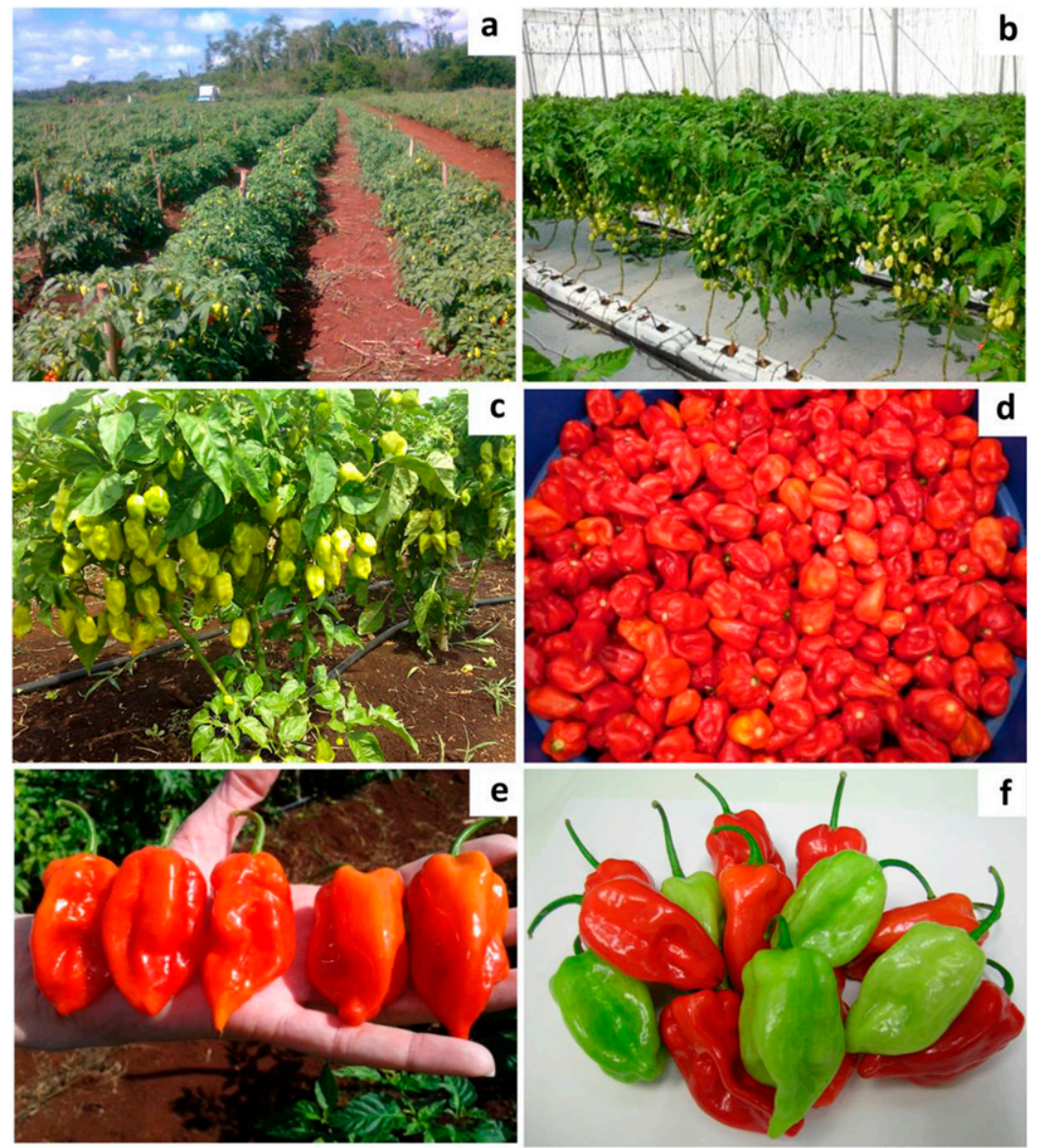

Fig. 1. Typical fruiting plants of the cultivar Mayan Kisin (A) field grown, (B) hydroponics, (C) greenhouse, (D and $\mathbf{E}$ ) observe the attractiveness, form, and dimensions of the fruit, (F) fruits at immature and mature stages.

by Collins et al. (1995), slightly modified by Canto-Flick et al. (2008), based on quantitative determinations, by means of high-performance liquid chromatography method.

'Mayan Kisin' displays upright growth and shortened internodes on the top. The transplant is performed at $45 \mathrm{~d}$ after sowing; days from transplanting to flowering are $45 \mathrm{~d}$. The first harvest of mature fruits can be carried out after $90 \mathrm{~d}$, and depending on the crop management, the harvest may remain for 6 months to a year. Anthocyanin pigmentation on the hypocotyl disappears as it grows. Pubescence is sparce on both stem and leaves. The leaves are medium, slightly blistered in the adaxial surface, oval shaped, and intense green color. Flowers are presented in the erect position; piquant. The majority of the fruits are triloculated, although fruits with four loculi (tetraloculated) with slightly deeper depressions can be observed, although less frequently.

Given its high productivity, strong pungency, attractions, and aromatic fruits, Mayan Kisin can be described as a "multipurpose" cultivar with a high potential to be used in the industry, for fresh consumption and for exportation.

The description was carried out in accordance with the descriptors published for capsicum (IPGRI, AVRDC, and CATIE, 1995) and habanero peppers (C. chinense Jacq.) (SAGARPA and SNICS, 2014) under greenhouse conditions.

\section{Availability}

Request for seeds should be directed to the Centro de Investigaciones Científicas de Yucatán (CICY), calle 43 no. 130, Chuburná de Hidalgo, Mérida, Yucatán 97200, México. Small seed samples are available for research purposes from Dr. N. Santana Buzzy, CICY, buzzy@cicy.mx; (999) 9428330, Ext. 226.

\section{Literature Cited}

Aguilar-Rincón, V.H., T. Corona Torres, P. López López, L. Latournerie Moreno, M. Ramírez Meraz, H. Villalón Mendoza, and J.A. Aguilar Castillo. 2010. Los chiles de México y su distribución. SINAREFI, Colegio de Postgraduados, INIFAP, ITA Conkal, UANL, UAN Montecillo, Texcoco, Estado de México. 114 p. 607-7533-68-9.

Canto-Flick, A., E. Balam-Uc, J.J. Bello-Bello, C. Lecona-Guzmán, D. Solís-Marroquín, S. Avilés-Viñas, E. Gómez-Uc, G. López-Puc, and N. Santana-Buzzy. 2008. Capsaicinoids content in habanero pepper (Capsicum chinense Jacq.): Hottest known cultivars. HortScience 43:1344-1349.

Collins, M.D., L.M. Wasmund, and P.W. Bosland 1995. Improved method for quantifying capsaicinoids in Capsicum using high-performance liquid chromatography. HortScience 30:137139.

IPGRI, AVRDC, and CATIE. 1995. Descriptors for Capsicum (Capsicum spp.). International Plant Genetic Resources Institute, Rome, Italy; the Asian Vegetable Research and Development Center, Taipei, Taiwan; and the Centro Agronómico Tropical de Investigación y Enseñanza, Turrialba, Costa Rica. ISBN 929043-216-0

Latournerie, L., J.L. Chávez, M. Pérez, C.F Hernández, R. Martínez, L.M. Arias, and G. Castañon. 2001. Exploración de la diversidad morfológica de chiles regionales en Yaxcabá, Yucatán, México. Agronomía Mesoamericana 12(1):41-47.

Royal Horticultural Society (RHS). 2001. The Royal Horticultural Society's colour chart. Royal Hort. Soc., London, UK.

SAGARPA and SNICS. 2014. Guía técnica para la descripción varietal de chile Habanero (Capsicum chinense Jacq.). Secretaría de Agricultura, Ganadería, Desarrollo Rural, Pesca y Alimentación, Servicio Nacional de Inspección y Certificación de Semillas, Estados Unidos Mexicanos. 